\title{
Validation of a food frequency questionnaire using 7 day weighed food diaries in an elderly population with arthritis
}

\author{
A. Aghili and D. Bhakta \\ Public Health Nutrition Research Group, Faculty of Life Sciences \& Computing, London Metropolitan University, \\ London $N 78 D B$
}

Currently there are 10 million people with symptoms of age-related arthritis in the UK, and there is continuing research in relieving symptoms of this condition with diet ${ }^{(1)}$. The Arthritic Association is a charity dedicated to supporting people with arthritis by helping them to manage their condition with diet and lifestyle advice. This involves the individual assessment and monitoring of dietary intake (using a 7 day food record) by a registered dietitian. The charity commissioned London Metropolitan University to develop and validate a new dietary assessment tool to assess absolute energy and macronutrient intake, which was less burdensome on an elderly population and could also be used in future research.

A semi-quantitative food frequency questionnaire (FFQ) was developed using a food list of common foods consumed by the membership from previously completed 7 day food records. The FFQ was piloted and then finalised with a total of 17 food groups, with an additional section for supplements and foods not listed. The self-administered FFQ, used household portion sizes was completed over a seven day period. The FFQ was validated against the reference measure of the 7 day weighed diary (7WD). The Arthritic Association advertised the study and asked for volunteers through their quarterly newsletter. Altogether 65 participants agreed to participate, of whom 60 completed the food frequency and 50 (83\% response rate) also completed the 7 day weighed diaries.

Nutrient intakes obtained from the FFQ were higher than those obtained from the 7WD (Table 1) although the level of underreporting was similar (7WD 30\%, FFQ 32\%). Spearman correlations for macronutrients and most micronutrients were within the acceptable range $r=0 \cdot 5-0 \cdot 7^{(2)}$ for both crude and energy-adjusted values. The ranking for the macronutrients ranged from $73-$ $88 \%$ within the same or adjacent quartile, with only $0-8 \%$ in the extreme quartile. Bland-Altman plots comparing the FFQ with the 7WD for energy, fat, protein, carbohydrate and fibre (NSP) were within acceptable limits of agreement ( \pm 1.96 SD). The newly developed FFQ was thus deemed a valid tool to assess dietary intake in this population.

Table 1. Spearman's correlation observed between the 7-day weighed diary and the FFQ

\begin{tabular}{|c|c|c|c|c|c|c|c|c|}
\hline & \multicolumn{2}{|c|}{$\begin{array}{l}\text { 7-d weighed diary } \\
n=50\end{array}$} & \multicolumn{2}{|c|}{ FFQ $n=50$} & \multicolumn{4}{|c|}{ Spearman's Correlation } \\
\hline & $\overline{\text { Mean }}$ & $S D$ & $\overline{M e a n}$ & $S D$ & $r$ & p-value & $r^{*}$ & p-value \\
\hline Energy $(\mathrm{kcal}) / \mathrm{d}$ & 1662 & $(488)$ & 1667 & (397) & 0.66 & $<0.001$ & & \\
\hline Protein $(\mathrm{g}) / \mathrm{d}$ & 71.6 & $(20 \cdot 8)$ & 71.7 & $(17 \cdot 1)$ & 0.43 & 0.002 & 0.59 & $<0.001$ \\
\hline Carbohydrate $(\mathrm{g}) / \mathrm{d}$ & 198 & (70) & 200 & $(54.5)$ & 0.67 & $<0.001$ & $0 \cdot 64$ & $<0.001$ \\
\hline Fat $(\mathrm{g}) / \mathrm{d}$ & 64.5 & (26) & $65 \cdot 3$ & (22) & 0.60 & $<0.001$ & 0.64 & $<0.001$ \\
\hline Saturated $(\mathrm{g}) / \mathrm{d}$ & $20 \cdot 9$ & $(10 \cdot 2)$ & 18.4 & $(6.9)$ & 0.60 & $<0.001$ & 0.51 & $<0.001$ \\
\hline Monounsaturated $(\mathrm{g}) / \mathrm{d}$ & $21 \cdot 3$ & $(9.4)$ & $23 \cdot 7$ & $(9.4)$ & 0.53 & $<0.001$ & 0.43 & 0.002 \\
\hline Polyunsaturated $(\mathrm{g}) / \mathrm{d}$ & $13 \cdot 3$ & $(7.0)$ & 15.9 & $(6 \cdot 3)$ & $0 \cdot 50$ & $<0.001$ & 0.45 & 0.001 \\
\hline Trans fatty acids $(\mathrm{g}) / \mathrm{d}$ & 1.3 & $(1.0)$ & 1.2 & $(0.7)$ & 0.33 & 0.02 & $0 \cdot 31$ & 0.03 \\
\hline $\mathrm{NSP}(\mathrm{g}) / \mathrm{d}$ & $16 \cdot 9$ & $(5 \cdot 4)$ & $19 \cdot 7$ & $(4 \cdot 3)$ & 0.41 & 0.03 & 0.43 & 0.02 \\
\hline Sodium $(\mathrm{mg}) / \mathrm{d}$ & 2052 & (789) & 2192 & (762) & $0 \cdot 36$ & 0.01 & $0 \cdot 15$ & NS \\
\hline Potassium $(\mathrm{mg}) / \mathrm{d}$ & 3165 & (1005) & 3410 & (760) & 0.56 & $<0.001$ & 0.57 & 0.57 \\
\hline Calcium $(\mathrm{mg}) / \mathrm{d}$ & 831 & (362) & 936 & (344) & 0.50 & $<0.001$ & $0 \cdot 40$ & 0.40 \\
\hline Magnesium (mg)/d & 319 & (95) & 354 & (93) & 0.54 & $<0.001$ & $0 \cdot 30$ & $0 \cdot 30$ \\
\hline Iron $(\mathrm{mg}) / \mathrm{d}$ & $12 \cdot 4$ & $(4 \cdot 3)$ & 12.7 & $(2.9)$ & $0 \cdot 41$ & $<0.001$ & $0 \cdot 27$ & $0 \cdot 27$ \\
\hline Vitamin $C(\mathrm{mg}) / \mathrm{d}$ & 116 & (90) & 144 & (59) & 0.62 & $<0.001$ & 0.68 & $<0.001$ \\
\hline Vitamin E (ug)/d & $9 \cdot 0$ & (3.9) & 11.2 & $(6 \cdot 3)$ & 0.50 & 0.002 & $0 \cdot 51$ & 0.002 \\
\hline Vitamin A $($ retinol $) / \mathrm{d}$ & 377 & (661) & 334 & (446) & 0.40 & 0.005 & 0.42 & 0.003 \\
\hline Vitamin D (ug)/d & $3 \cdot 8$ & $(2 \cdot 4)$ & $3 \cdot 1$ & $(1 \cdot 3)$ & $0 \cdot 17$ & NS & 0.41 & 0.003 \\
\hline
\end{tabular}

$r^{*}$ adjusted for energy, NS not significant

1. http://www.arthritisresearchuk.org/ (accessed 270314).

2. Willett W (1998) Nutrition Epidemiology $2^{\text {nd }}$ edition. New York. Oxford University Press. 